\title{
Globale Partizipation der Zivilgesellschaft und indigene Völker
}

INFOE steht für „Institut für Ökologie und Aktions-Ethnologie“, eine Organisation, die 1987 gegründet wurde und die in Deutschland die erste ihrer Art war, die indigene Völker (Ureinwohner/innen) weltweit bei der Verteidigung ihrer natürlichen Lebensgrundlagen und Menschenrechte unterstützt. Von Anfang an war INFOEs Ansatz also ein globaler. Es ging und geht um das Aufdecken und Aufzeigen weltweiter Zusammenhänge: „Was hat eine Coladose mit dem tropischen Regenwald zu tun?" war die Leitfrage einer unserer ersten Ausstellungen und die Antwort verwies auf die Mitverantwortung der europäischen Verbraucher/-innen.

Mit dem 500sten Jahrestag des Beginns der Kolonisierung Amerikas 1992 bekam das Thema indigene Völker so viel Aufmerksamkeit in der Öffentlichkeit wie davor und danach nicht mehr. In der deutschen Fachöffentlichkeit setzte sich die Erkenntnis durch, dass die 350 Millionen Angehörigen indigener Völker aller Kontinente die Hauptleidtragenden des weltweiten Hungers nach Erdöl, Erdgas, Uran, Kohle und anderen Rohstoffen sind. Auf der Berliner Strahlenopfer-Konferenz und beim World Uranium Hearing in Salzburg berichteten Angehörige der Hopi Nordamerikas, der Nenzen aus der russischen Arktis, der australischen Aborigines, und dutzender anderer indigener Völker aller Kontinente einer erschütterten Fachöffentlichkeit davon, wie es an jedem Glied der nuklearen Kette - Uranbergbau, Uranverarbeitung, Atomtests, Lagerung von Nuklearabfall - fast immer indigene Völker trifft. Obwohl sie nur fünf Prozent der Weltbevölkerung ausmachen, sind sie rund vier Fünftel der Betroffenen. Und in anderen Sektoren - Erdöl, Großstaudämme, Bergbau etc. sieht es ähnlich aus.

Indigene Völker - eine globale Bewegung Waren anfänglich noch Briefpost, Faxgerät und Telefon die Kommunikationsmittel der Wahl, veränderte die Verbreitung des Internets in universitären und NGO-Kreisen zu Beginn der 90er Jahre alles. Plötzlich waren die Indigenen nicht nur sehr nahe gerückt, sondern als globale Bewegung, die wir seitdem nach Kräften unterstützt haben, greif- und erfahrbar geworden. Seit den 80er Jahren schon waren Indigene regelmäßige Teilnehmer der UN-Arbeitsgruppe für indigene Bevölkerungen (damals mieden die Staaten noch nach Kräften das Wort, Völker'/,peoples', aus dem indigene Völker das Recht auf Selbstbestimmung ableiten). Sie verhandelten in UN-Institutionen beharrlich über einer UN-Erklärung der Rechte indigener Völker, die nach einem Vierteljahrhundert Verhandlung 2007 endlich verabschie- det wurde. Sie stritten für die Einrichtung eines ständigen UN-Forums für indigene Angelegenheiten; sie wurden aktive Unterhändler bei den UN-Klimaverhandlungen und den Verhandlungen um die Biodiversitätskonvention. So ist mit den Jahren die offizielle Anerkennung indigener Völker auf zwischenstaatlicher und internationaler Ebene immer weitergewachsen; mit dem UN-Gipfel über indigene Völker 2014 als vorläufigen Gipfelpunkt, wo erstmals Vertretende indigener Völker an einer UN-Generalversammlung teilnehmen durften. Mittlerweile ist ihre Beteiligung an internationalen Prozessen als eigene Gruppe neben Staaten und NGOs die Regel und nicht mehr die Ausnahme. INFOE hat seit mittlerweile anderthalb Jahrzehnten ihre Teilnahme an diesen Prozessen regelmäßig praktisch unterstützt, gemeinsam mit anderen NGOs, die der überschaubaren Community der Unterstützer indigener Völker angehören. Immer ist dabei selbstverständlich, dass INFOE zwar mit Rat und Tat zur Seite steht, sich aber nicht herausnimmt für die Indigenen zu sprechen, wie dies allzu oft in der Geschichte der Fall war.

\section{Anerkennung indigener Rechte - Lippenbekenntnis oder Realität?}

Auf internationaler Ebene sind essenzielle Forderungen indigener Völker, wie etwa die Anerkennung des Umstands, dass Projekte, die ihre Territorien betreffen, ihre freie, vorherige und informierte Zustimmung (Free, Prior and Informed Consent, FPIC) brauchen weitgehend anerkannt, selbst Verbände der Öl- und Bergbauindustrie - unser „Hauptgegner“ für viele Jahrzehnte - erkennen es mittlerweile teilweise an.

Doch eine komplett andere Frage ist es, ob und wie im konkreten Fall solche Rechtsprinzipien umgesetzt werden. Daher ist es nicht allein damit getan, wenn von internationalen Organisationen und Industrieverbänden wohlfeile Erklärungen und Policies verabschiedet werden. Zwar haben mittlerweile praktisch alle großen Lebensmittelkonzerne FPIC in ihren Policies anerkannt, doch als wir 2016 die UN-Arbeitsgruppe für Wirtschaft und Menschenrechte bei der Erarbeitung eines Berichts unterstützten, der den Zucker- und Palmölsektor in Augenschein nahm, stellte sich heraus, dass die UN bisher kaum Mittel geschaffen haben, um die Einhaltung dieser Policies einzufordern und zu kontrollieren. Selbst wenn Staaten FPIC in der Gesetzgebung festgeschrieben haben, ist die Praxis oftmals, dass man einfach versucht, Vertreterinnen und Vertreter der jeweiligen Gemeinschaft mit Versprechungen oder Dro- 
hungen eine Unterschrift abzuluchsen, mit der man dann anschließend die Lizenz für alles zu haben glaubt, oft im Tausch für den Bau von Schulen oder Straßen - Aufgaben, die eigentlich der Staat ohnehin zu erfüllen hätte.

Die entscheidenden Auseinandersetzungen finden also nach wie vor vor Ort statt - dort wo indigene Gemeinschaften leben, dort wo sie konkret um die Wahrung ihrer Land- und Menschenrechte kämpfen. Auch hier sind INFOE und seine Partner als Verbündete und Unterstützer tätig. So wie bei den Wampis in Peru, den Jurana in Brasilien, den Subanon auf den Philippinen und den Embera Chami in Kolumbien, vier indigene Völker, die ihre eigenen Regeln und Protokolle dafür entwickelt haben, wie Freie, Vorherige und Informierte Zustimmung auszusehen hat. Gewalt und Druck zum Trotz beharren sie darauf, dass Konzerne und Regierungen ihre Regeln anerkennen müssen, wenn sie mit ihnen verhandeln wollen. Dazu gehört natürlich auch das Recht, am Ende einer Verhandlung doch „Nein“ zu sagen oder sich eine Verhandlung gegen den eigenen Willen nicht aufzwingen zu lassen.

Auseinandersetzungen finden auf allen Kontinenten statt, in Entwicklungs- wie auch in Industrieländern - wie der Widerstand gegen die Dakota-Access-Pipeline und die Keystone-XL-Pipeline durch das Gebiet, das laut dem Vertrag von Fort Laramie von 1868 zwischen den Lakota/Sioux und den Vereinigten Staaten auf immer der Great Sioux Nation gehören sollte. 2015 machte der Widerstand weltweit Schlagzeilen, als sich trotz massiver Polizeigewalt Angehörige hunderter India- nerstämme aus ganz Nord- und Südamerika dem Widerstand anschlossen und das Projekt zunächst zu stoppen schienen aber dann mit der unerwarteten Wahl Trumps erleben mussten, wie sich Staat und Konzerne ein weiteres Mal straflos über ihre Rechte hinwegsetzen. Doch trotz aller Rückschläge geht der Widerstand beharrlich weiter.

In anderen Teilen der Welt ist die Lage nicht minder dramatisch, etwa in den Bergbau-, Öl- und Gasfördergebieten Russlands, von wo Deutschland den Großteil seiner Rohstoffe bezieht, und das mit erheblicher Unterstützung des deutschen Staates und tatkräftiger Lobbyarbeit von Ex-Kanzler Gerhard Schröder. Im autoritären Russland Putins gibt es jedoch kaum offene Proteste von Indigenen, sodass die dortige Realität nur sehr selten Schlagzeilen macht. Hier beharrlich am Ball zu bleiben und dicke Bretter zu bohren, auch wenn die Lage wenig hoffnungsvoll erscheint und das Thema gerade keine Konjunktur hat - das ist es, was echte Partner ausmacht. Die Perspektive indigener Völker bemisst sich in Jahrhunderten. Auch wir sollten verstehen, dass wir das, was wir tun, in erster Linie für die Generationen tun, die nach uns kommen.

Johannes Rohr doi.org/10.31244/zep.2019.03.06

\section{UNSERE BUCHEMPFEHLUNG}

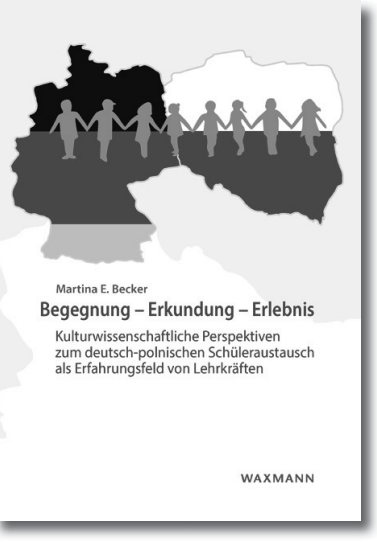

2019, 430 Seiten, br., 44,90€, ISBN 978-3-8309-4011-1

E-Book: 39,99€, ISBN 978-3-8309-9011-6
Martina E. Becker

\section{Begegnung - Erkundung - Erlebnis}

Kulturwissenschaftliche Perspektiven zum deutsch-polnischen Schüleraustausch als Erfahrungsfeld von Lehrkräften

Interkultureller Kontakt zeigt sich derzeit angesichts der vielfältigen globalen Entwicklungen als mannigfacher Diskussionsgegenstand. Auch die deutsch-polnischen Beziehungen unterlagen in den letzten Jahrzehnten unterschiedlichen Annäherungs- und Entfernungsstadien. Der Blick auf Lehrkräfte, die maßgeblich für Austauschprojekte an Schulen dieser beiden Länder seit den 1970er Jahren verantwortlich zeichnen, fehlte jedoch bisher in der volkskundlich-kulturanthropologischen Forschung. 